\title{
Reviewed immunosensor format using nanomaterial for tungro virus detection
}

\begin{abstract}
Recently, nanomaterial based biosensor application has drawn a lot attention among researchers because of specialty to enhance the sensor signal for increasing the sensitivity for detecting and identification of pathogen, viruses and toxic compound in controlling plant disease outbreak effectively. Rice tungro disease (RTD) causes a major problem in rice production and also will effect in the economic loss in the country. Therefore, early detection system is needed to monitor the disease at the early stage of the infection for preventing the disease outbreak in planting area. Lastly, this paper will discuss the current findings in rapid diagnostics using immunosensors technologies with nanomaterial application in enhancing the sensor signal for increasing the detection sensitivity.
\end{abstract}

Keyword: Immunoassay; Immunosensor; Nanomaterial; Tungro virus 